\title{
Violent expansion of a rising Taylor bubble
}

\author{
Guangzhao Zhou* and Andrea Prosperetti ${ }^{\dagger}$ \\ Department of Mechanical Engineering, University of Houston, Houston, Texas 77204, USA
}

(Received 26 December 2018; published 16 July 2019)

\begin{abstract}
This paper studies the rise of a Taylor bubble in a long, vertical liquid-filled conduit such as the riser of an oil production or exploration well. It is shown that, as the bubble rises and expands due to the gradually decreasing hydrostatic pressure, a situation may be reached in which the character of the expansion abruptly transitions from moderate to very violent. The phenomenon can be particularly dangerous with very deep wells, in which a "gas kick," i.e., the intrusion of gaseous hydrocarbons in the riser, can result in a violent and destructive "blowout." As an example, with its expansion, a 1-m-long bubble rising in a 1000-m-long tube causes the liquid to spill out of the top of the tube with a velocity of the order of $\mathrm{cm} / \mathrm{s}$ for the better part of an hour, until it rises to the order of $10 \mathrm{~m} / \mathrm{s}$ in the last several seconds. This process is considered to have been responsible for the DeepWater Horizon accident in the Gulf of Mexico in 2010. The same phenomenon underlies volcanic eruptions of the Strombolian type, which are characterized by the intermittent ejection of material up to heights of tens or even hundreds of meters. In this paper the process is studied with a quasiequilibrium model, a dynamic model, and a drift-flux model, all of which give results in substantial agreement with each other. The quasiequilibrium model leads to a very simple relation which, while approximate, gives a robust criterion for the occurrence of violent bubble expansions. The results of an illustrative calculation with OpenFOAM are also presented.
\end{abstract}

DOI: 10.1103/PhysRevFluids.4.073903

\section{INTRODUCTION}

During drilling, completion, and production activities of an oil well, it is possible that formation gas enters the riser in the form of a large Taylor bubble creating a gas-in-riser event. The formation of a large bubble can also occur as gas dissolved in the drilling fluid, especially of the oil-based type, comes out of solution due to the decreasing hydrostatic pressure. If the presence of this gas remains undetected, then an extremely dangerous "riser gas blowout" can take place, in which hydrocarbons are expelled from the top of the riser at an exceedingly fast rate. If this flammable material spills into a confined environment in which ignition sources are present, then a very destructive explosion can take place. The phenomenon is particularly insidious because transition from spilling velocities of the order of $\mathrm{cm} / \mathrm{s}$ to velocities two orders of magnitude larger can take place in a few seconds, thus rendering the adoption of mitigating measures virtually impossible. Such a sequence of events is considered to have been the cause of the disastrous DeepWater Horizon accident in the Gulf of Mexico in 2010 [1].

Over the past few decades several well-control simulators have been developed in the oil industry, mostly relying on models of the drift-flux type similar to the one used below in Sec. V (see, e.g.,

\footnotetext{
*gzhou5@uh.edu

Also: Faculty of Science and Technology and J. M. Burgers Center for Fluid Dynamics, University of Twente, 7500AE Enschede, The Netherlands; aprosperetti@uh.edu
} 
Refs. [2,3]). Motivated by the new developments in exploration and production in deep and ultradeep waters, the more recent work in this area has focused on the phenomenon of present interest, recognizing the possibility of the abrupt transition from a mild to a violent expansion of the rising bubble (see, e.g., Refs. [4,5]). In particular, the examples given in Ref. [4] show the rapid expansion of the Taylor bubble but the paper does not determine the conditions under which the phenomenon takes place. A valuable contribution of this work is the explicit consideration of the ex-solution of dissolved gas which is shown to lead a sequence of events similar to that of an initially fully formed Taylor bubble.

The literature devoted to the physics of Taylor bubbles contains studies of the expansion of rising bubbles due to the falling hydrostatic pressure (see, e.g., Refs. [6,7]). As will be explained in Sec. II below, however, these experiments were conducted in a parameter range in which violent expansions were not possible and, indeed, none was reported.

Another process in which the phenomenon studied here plays an important role is volcanic eruptions of the so-called Strombolian type (see, e.g., Refs. [8-13]). Citing from Ref. [8], on the Italian island of Stromboli, "During normal activity, the rise and bursting of large gas slugs at the surface of the magma column cause recurring explosive events, which last tens of seconds and have a return interval of a few minutes (5-20 events per hour)... These explosions result in the emission of jets of gas and incandescent magma fragments to heights of 100-200 m above the vents... The current volcanic activity at Stromboli has persisted, without a significant break or change in style, for at least the last 1300 years."

The work reported in Ref. [10] was directed to the study of Strombolian eruptions and is among the first experimental and theoretical investigations of the rapid expansion of Taylor bubbles. The authors used a relatively high-viscosity oil (viscosity $0.124 \mathrm{~Pa} \mathrm{~s}$ ) to approximate magma and carried out experiments in a 2-m-long tube. They realized that, to observe the rapid expansion in such a short tube, it was necessary to reduce the pressure on the free surface at the top of the tube, which they were able to lower down to $1 \mathrm{~Pa}$. They report several observations of the rapid expansion of the Taylor bubble which they were able to compare fairly successfully with a simple model (considered further below in Sec. III) and with fully three-dimensional Navier-Stokes simulations. Their later publication [11] is devoted to an understanding of the conditions under which, instead of the violent bubble expansion, the gas is emitted from the vent in a mode that volcanologists refer to as "puffing," namely the mild venting of the bubble at the crater free surface. The criterion that they propose is very similar to the one shown below in Eqs. (11) and (13), which demonstrates in very simple terms the importance of the initial bubble depth and of the pressure above the liquid free surface.

The purpose of the present paper is, first, to give a physically transparent explanation of the phenomenon of violent bubble expansion and, second, to show its very robust nature by proving that essentially the same result is found with a variety of models. This near-coincidence of results supports the validity of the general understanding of the phenomenon described. We begin with a quasiequilibrium balance of forces in Sec. II, followed by a dynamic model based on controlvolume balances in Sec. III, and a drift-flux model in Sec. V. Section VI shows how the drift-flux computation can be made much more efficient by adopting a moving reference frame rising with the bubble. The fact that the simple dynamic model, based on a single ordinary differential equation, gives results quite comparable to the more complex drift-flux model is an interesting result as it permits to carry out extensive exploratory calculations at a fraction of the computational cost of the drift-flux model.

\section{A QUASIEQUILIBRIUM MODEL}

In this section we use a very simple model to explain the physics responsible for the rapid bubble expansion at the root of the gas-in-riser phenomenon. The subsequent sections show that, in spite of its rather drastic simplifications, the model gets to the core of the process.

We consider a vertical tube and, in the simplest version of the model, approximate a rising Taylor bubble as a section of this tube filled of gas. The pressure at the top end of the tube is kept constant 

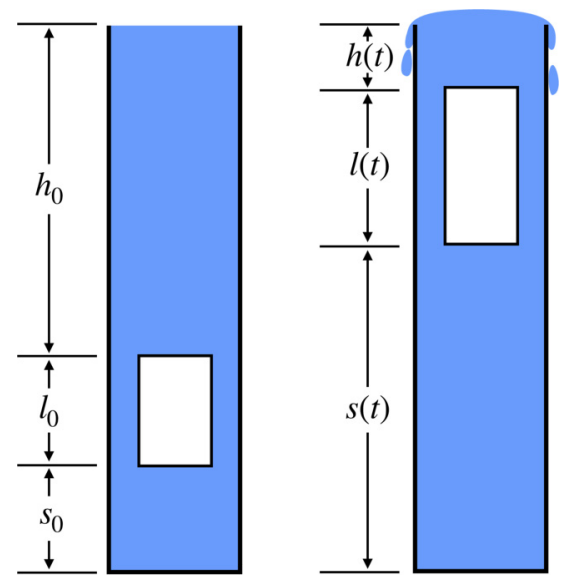

FIG. 1. Sketch of the situation considered in the quasiequilibrium model of Sec. II and some nomenclature. Initially the liquid free surface is at the top of the tube (left, $t=0$ ) so that the liquid spills out as the bubble expands (right, $t>0$ ).

at a value $p_{t}$, which could be the atmospheric pressure if the tube is open, whereas the bottom end of the tube is closed. At time $t$, the bubble has a length of $\ell(t)$. Its upper surface is located at a depth $h(t)$ below the liquid surface at the top of the tube and its bottom surface is at a height $s(t)$ above the tube bottom. We assume that, initially, the liquid level in the tube is at the same height as the tube top so that, as the bubble expands, liquid spills out and the liquid level remains at the same position (Fig. 1). Later in this section and in the following one we will also consider the case in which the tube continues above the initial position of the liquid so that the free surface is pushed up as the bubble expands (Fig. 3, right).

The instantaneous gas pressure in the bubble is $p_{g}(t)$. If we approximate the gas as perfect and isothermal then, for a given gas content, and with the neglect of dynamical effects, the volume (or length $\ell$, if the bubble radius remains constant) of the bubble is dictated by the pressure balance,

$$
p_{g}=p_{g 0} \frac{\ell_{0}}{\ell}=p_{t}+\rho g h
$$

where the index 0 denotes conditions at the initial instant, $\rho$ is the liquid density, and $g$ is the acceleration of gravity. Clearly, $p_{g 0}=p_{t}+\rho g h_{0}$ (see Fig. 1) so that

$$
\frac{p_{g 0}}{\rho g}=\frac{p_{t}}{\rho g}+h_{0} \equiv h_{t}+h_{0},
$$

with $h_{t}=p_{t} / \rho g$ the head corresponding to the pressure acting on the liquid free surface at the top of the tube.

Upon taking the time derivative of Eq. (1) we find

$$
-p_{g 0} \frac{\ell_{0}}{\ell^{2}} \dot{\ell}=\rho g \dot{h}
$$

with superposed dots denoting time derivatives. The rate of change of the bubble length is just the difference between the velocity $u_{T}$ of its top surface and the velocity $u_{B}$ of its bottom surface:

$$
\dot{\ell}=u_{T}-u_{B} .
$$

If the liquid spills out of the tube so that its level remains at the same height whatever the bubble volume, as we have assumed, then the sum of the length of tube occupied by the liquid, $s+h$, and 
of the bubble length, $\ell$, equals the constant tube length so that $s+\ell+h=$ const., from which

$$
\dot{h}=-u_{B}-\left(u_{T}-u_{B}\right)=-u_{T},
$$

as expected. The derivation of this relation presupposes that $u_{B}$ remains constant as the bubble rises. This assumption underlies the original theories of Refs. [14] and [15], where it was referred to the bubble nose rather than its base. However, it is recognized in the literature that the velocity of the top of an expanding bubble is larger than that of the bottom (see, e.g., Refs. [7,16,17]), the implication being that the latter is constant. This fact was observed experimentally and explicitly stated in Ref. [10].

Upon substituting Eqs. (4) and (5) into Eq. (3) we find

$$
\frac{u_{T}}{u_{B}}=\frac{1}{1-\ell^{2} / \ell_{c}^{2}},
$$

where

$$
\ell_{c}=\sqrt{\frac{p_{g 0}}{\rho g} \ell_{0}}=\sqrt{\left(h_{t}+h_{0}\right) \ell_{0}}
$$

is a critical bubble length as is evident from the fact that $u_{T} / u_{B} \rightarrow \infty$ as $\ell \rightarrow \ell_{c}$. Clearly, the present quasiequilibrium model becomes inapplicable after this point find

We can better understand this result by using Eq. (6) to express $u_{T}$ in Eq. (4) and integrating to

$$
-\frac{\ell_{c}^{2}}{\ell}+\frac{\ell_{c}^{2}}{\ell_{0}}-\ell+\ell_{0}=u_{B} t=s(t)-s_{0},
$$

where, as before, we have taken $u_{B}$ to be a constant. By substituting $\ell=\ell_{c}$ in this relation we find the height $s_{c}$ reached by the bottom of the bubble when its length becomes equal to $\ell_{c}$ :

$$
s_{c}=\ell_{0}-2 \ell_{c}+\frac{\ell_{c}^{2}}{\ell_{0}}+s_{0} .
$$

For the critical situation embodied in this result to actually occur, it is evidently necessary that the bubble top still be below the top of the tube, i.e., that $s_{c}+\ell_{c} \leqslant \ell_{0}+h_{0}+s_{0}$. Substituting Eq. (9) into this relation and using Eq. (7) to eliminate $\ell_{c}$, we obtain

$$
\sqrt{\left(h_{t}+h_{0}\right) \ell_{0}} \geqslant h_{t}
$$

or

$$
\Gamma \equiv\left(1+\frac{h_{0}}{h_{t}}\right) \frac{\ell_{0}}{h_{t}} \geqslant 1 .
$$

This parameter is close to a similar quantity defined in Ref. [8], and it can be interpreted as the dimensionless length of the bubble expanded from the initial pressure $p_{t}+\rho g h_{0}$ to the pressure $p_{t}$. It can also be readily shown that $h_{c} / h_{t}=\sqrt{\Gamma}-1$, where $h_{c}$ is the depth of the liquid column at the time when $\ell$ reaches $\ell_{c}$ [8]. An interpretation which perhaps has a greater physical relevance is that, for an isothermal process, $\Gamma$ is a dimensionless measure of the initial enthalpy of the bubble $\left(p_{t}+\rho g h_{0}\right) A_{b} \ell_{0}$, with $A_{b}$ the cross section of the bubble.

The relation Eq. (11) is shown graphically in Fig. 2. The line marks the locus along which the two sides of Eq. (11) are equal, which corresponds to the bubble length $\ell$ reaching the critical value $\ell_{c}$ when the top of the bubble is just at the top of the tube. The region above the line corresponds to parameter values such that $\ell$ approaches $\ell_{c}$ while the bubble is still submerged in the tube. Equation (6) shows that, when this happens, a catastrophic expulsion of liquid from the top of the tube with a very high velocity will take place. The region below the line corresponds to the parameter range in which $\ell$ never approaches $\ell_{c}$ in its rise. 


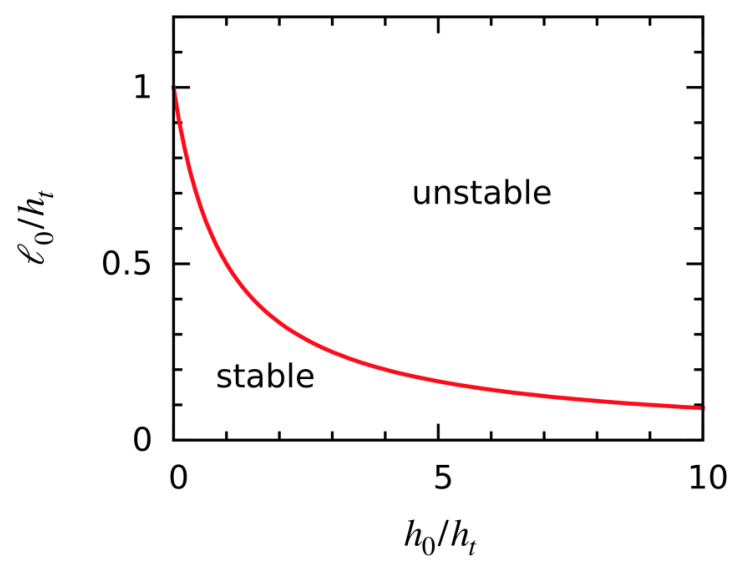

FIG. 2. Graphical representation of the stability boundary Eq. (11) $\Gamma=1 ; h_{0}$ and $\ell_{0}$ are the initial depth and length of the bubble; $h_{t}=p_{t} /(\rho g)$, with $p_{t}$ the pressure at the top of the tube, is the head at the top of the tube. For initial conditions above the curve the critical length $\ell_{c}$ given by Eq. (7) is reached as the bubble rises in the tube, which marks the onset of the uncontrollable expansion of the bubble. This process is illustrated in the figures that follow and, in particular, in Fig. 8.

The form of Eq. (11) is particularly simple and easy to use and clearly demonstrates the increasing likelihood of a violent expansion as the initial submergence of the bubble $h_{0}$ increases beyond the hydrostatic head $h_{t}$, and as the initial bubble length $\ell_{0}$ increases. It will be seen in the subsequent sections that, in spite of the idealized derivation, this relation gives a good estimate of the transition from a gradual to a rapid, uncontrollable expansion.

The experiments of Ref. [7] were conducted in the range $h_{0} / h_{t}<0.4, \ell_{0} / h_{t}<0.05$, in which no rapid expansion is expected and, indeed, it was not reported by the authors. The later experiments reported in Ref. [6] correspond to $\ell_{0} / h_{t}<0.25, h_{0} / h_{t}<2.25$, much closer to the critical curve but still below it. The authors noticed a more significant expansion, but again without the rapid phase.

The model can be refined by allowing for the bubble to have the shape of cylinder with a (constant) cross section $A_{b}$ smaller than the cross section $A$ of the tube as sketched in Fig. 1. In this case, for the same condition as before in which the initial liquid level is at the mouth of the tube, Eq. (5) remains valid since the sum $s(t)+l(t)+h(t)$ must remain constant and equal to the total tube length.

Whether $A_{b}=A$ or $A_{b}<A$, however, makes a difference if the tube extends past the initial liquid level so that, as the bubble expands, the liquid is pushed upward in the tube rather than spilling out of it (Fig. 3, right); this is the situation studied both theoretically and experimentally in Refs. [10,11]. In this case the liquid mass is conserved so that, with a constant $A_{b}, A s(t)+\left(A-A_{b}\right) \ell(t)+A h(t)$ remains constant. Upon differentiating we then find

$$
\dot{h}=-(1-\eta)\left(u_{T}-u_{B}\right)-u_{B}
$$

with $\eta=A_{b} / A$. This result contains Eq. (5) as a special case when $\eta=0$. (Of course, this is only a formal analogy and does not imply $A_{b}=0$.) The previous analysis can be carried out in this case as well finding, in place of Eq. (7),

$$
\ell_{c}=\sqrt{\frac{\left(h_{t}+h_{0}\right) \ell_{0}}{1-\eta}} .
$$



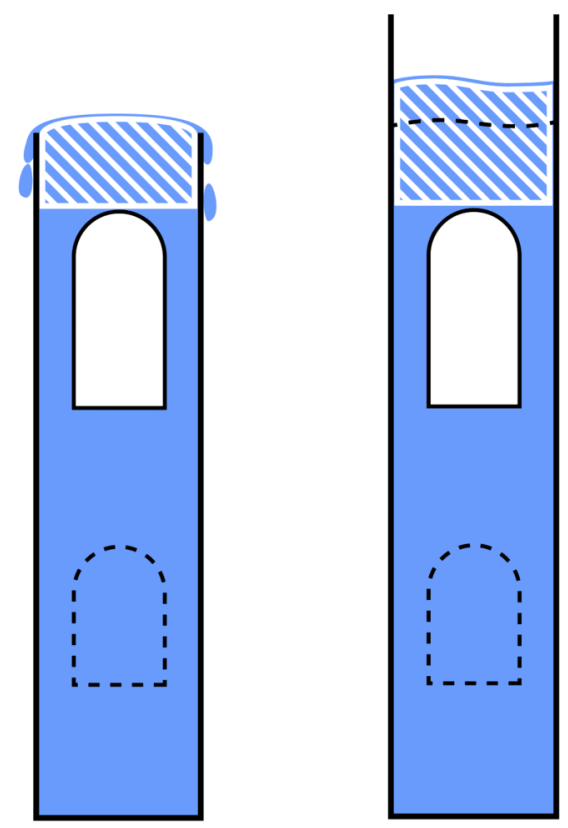

FIG. 3. The hashed region shows the control volume used with Eqs. (16) and (17): the lower boundary is a cross section of the tube tangent to the bubble top; the upper boundary is the liquid free surface, which remains at the tube top in the case sketched on the left, while it rises as the bubble expands in the case on the right. The dashed lines denote the initial positions of the bubble and of the liquid free surface.

As $\eta \rightarrow 1, \ell_{c}$ grows without bound. The time $t_{c}$ necessary for $s$ to equal $s_{c}$ can be calculated from Eq. (8) by setting $s=s_{c}$ or from its analog when $\eta \neq 0$; the result is

$$
t_{c}=\frac{1-\eta}{u_{B}}\left(\ell_{0}-2 \ell_{c}+\frac{\ell_{c}^{2}}{\ell_{0}}\right) .
$$

The same analysis leading to Eqs. (10) and (11) can be repeated with the result

$$
\frac{\sqrt{(1-\eta)\left(h_{t}+h_{0}\right) \ell_{0}}}{h_{t}} \geqslant 1 \text {. }
$$

This relation agrees with that given in Ref. [11]. When plotted as in Fig. 2, the stability boundaries move up as $\eta$ increases from zero. Satisfying this condition requires therefore either a larger and larger initial submergence or a larger and larger initial bubble length as the thickness of the liquid film surrounding the bubble decreases. The implication is that the length of tube necessary to observe a rapid bubble expansion also increases. Since, for most Taylor bubbles, $1-\eta$ tends to be small, this result implies that, as they rise in a tube extending above the initial liquid level, bubbles will approach the critical length only in relatively long tubes. The more frequent and realistic case in which the initial liquid level is at the tube mouth corresponds to $\eta=0$ : the previous analysis applies irrespective of the bubble cross-sectional area and, all other things being equal, results in the shortest tube length necessary for the violent expansion to occur.

\section{A SIMPLIFIED DYNAMIC MODEL}

The previous model neglects all dynamical effects such as the liquid inertia and the pressure drop due to viscosity and turbulence. We can refine the model by incorporating these effects in a simplified form. 
The starting point is the integral form of the momentum equation in the upward vertical direction $z$ :

$$
\frac{d}{d t} \int_{V} \rho u d V=\oint_{S} \rho u(\mathbf{v}-\mathbf{u}) \cdot \mathbf{n} d S-\oint_{S} p n_{z} d S+\oint_{S}(\tau \cdot \mathbf{n})_{z} d S-\int_{V} \rho g d V .
$$

Here $V$ is the control volume shown in Fig. 3, to be specified presently, $S$ is its complete boundary, $u$ is the vertical component of the liquid velocity $\mathbf{u}, \mathbf{v}$ is the local velocity of the interface $S, \mathbf{n}$ is the outward unit normal, $p$ is the pressure, $\tau$ is the sum of the viscous and Reynolds stresses, and the subscript $z$ denotes the vertical component. This equation is complemented by the analogous statement for the conservation of mass of an incompressible fluid:

$$
\frac{d}{d t} \int_{V} \rho d V=\oint_{S} \rho(\mathbf{v}-\mathbf{u}) \cdot \mathbf{n} d S
$$

The control volume to be used with these two relations is chosen as shown in Fig. 3: it is a section of tube bounded below by a horizontal cross-section tangent to the bubble nose and above by the free surface of the liquid in the tube which, as before, can remain at a fixed height if the initial liquid level is at the tube mouth (Fig. 3, left), or rise as the bubble expands if the tube continues past the initial liquid level (Fig. 3, right).

For the left-hand side of the mass balance Eq. (17) we simply have

$$
\int_{V} \rho d V \simeq A \rho h(t)
$$

where $h(t)$ is the height of the liquid above the bubble nose. For the right-hand side we have contributions from the top and bottom of the control volume. For the latter, $v_{z}=-u_{T}$ and we write this contribution as $-\rho A\left(u_{T}-\bar{u}\right)$, with $\bar{u}$ an average velocity over the lower boundary of the control volume. For the upper surface we must distinguish between the two cases that we consider. If the liquid spills out of the tube, $v_{z}=0$ and $(v-u)_{z} \simeq-\tilde{u}$, with $\tilde{u}$ the average velocity at the tube top. If, however, the liquid level rises in the tube, then $(v-u)_{z}=0$. To carry both cases at the same time we write

$$
\oint_{S}(\mathbf{v}-\mathbf{u}) \cdot \mathbf{n} d S=-A \alpha \tilde{u}-A\left(u_{T}-\bar{u}\right)
$$

where $\alpha=0$ or $\alpha=1$ according as to whether the tube continues above the initial liquid level or not. With the same convention, clearly

$$
\dot{h}=(1-\alpha) \tilde{u}-u_{T},
$$

so that, upon substitution into Eq. (17), we find $\tilde{u}=\bar{u}$ as expected, independently of $\alpha$. In view of this result, we will not distinguish between $\tilde{u}$ and $\bar{u}$ in the following and we will assume that they equal the volume-averaged velocity $u$ in the control volume.

For the momentum Eq. (16) we start by setting

$$
\int_{V} \rho u d V \simeq \rho u A h(t)
$$

For the first term in the right-hand side we have

$$
\oint_{S} u(\mathbf{v}-\mathbf{u}) \cdot \mathbf{n} d S \simeq-\alpha u^{2} A-u\left(u_{T}-u\right) A .
$$

Motivated by the continuity of pressure across the bubble surface we approximate the liquid pressure over the lower surface of the control volume by the pressure $p_{g}$ in the bubble so that

$$
\oint_{S} p n_{z} d S=\left(p_{t}-p_{g}\right) A
$$


In the viscous/Reynolds stress term we neglect the contribution of the bases of the control volume vis-a-vis that of the lateral surface, as is normally done, writing

$$
\oint(\tau \cdot \mathbf{n})_{z} d S \simeq-\pi d h \tau_{w},
$$

with $d$ the inner diameter of the tube and $\tau_{w}$ the wall shear stress, which can be expressed in terms of the Fanning friction factor,

$$
f=\frac{\tau_{w}}{\frac{1}{2} \rho u^{2}}
$$

For laminar flow in a round tube,

$$
f=\frac{16}{\operatorname{Re}_{d}},
$$

with $\operatorname{Re}_{d}=u d / v$ the Reynolds number based on the tube diameter and $v$ the liquid kinematic viscosity. An expression that well approximates $f$ in the case of turbulent flow for Reynolds numbers up to $10^{5}$ is given by Blasius's formula as

$$
f=0.079 \mathrm{Re}_{d}^{-1 / 4} .
$$

Finally,

$$
\int_{V} \rho g d V=\rho g A h
$$

Upon collecting the various contributions we have

$$
\rho A \frac{d}{d t}[u h(t)] \simeq-\alpha \rho u A-\rho u\left(u_{T}-u\right) A-\left(p_{t}-p_{g}\right) A-\pi d h \tau_{w}-\rho g A h,
$$

or, upon using Eq. (20) to express $\dot{h}$,

$$
A h \rho \frac{d u}{d t}=-\left(p_{t}-p_{g}\right) A-\rho g A h-\pi d h \tau_{w},
$$

or

$$
\rho h \frac{d u}{d t}=p_{g}-\left(p_{t}+\rho g h\right)-4 \frac{h}{d} \tau_{w} .
$$

Equations (20) and (31) contain the four unknowns $p_{g}, h, u$, and $u_{T}$ and therefore two additional relations are necessary to close the system. The gas pressure can be found from the first equality in Eq. (1). This relation introduces the new unknown $\ell$ which can be found from Eq. (4). The last equation can be derived from the balance equation for the entire liquid volume. At any instant the liquid volume in the tube is

$$
A s_{0}+A u_{B} t+\left(A-A_{b}\right) \ell+A h
$$

in which $s_{0}$ is the height of the liquid below the bubble base at the initial instant, $u_{B} t$ is the distance traveled by the bubble base with the constant velocity $u_{B}$ during the time $t$, and the next two terms are the liquid volume between the base and the tip of the bubble, with the last term the liquid volume in the control volume. This amount of liquid is constant if the tube extends beyond the initial liquid level, while it equals the initial liquid volume minus $A \int_{0}^{t} u(t) d t$, the total amount of liquid spilled, if the tube exit coincides with the initial liquid free surface. Upon taking the time derivative and using Eq. (20) to express $\dot{h}$ we have

$$
A u_{B}+\left(A-A_{b}\right) \dot{\ell}+A(1-\alpha) u-A u_{T}=-\alpha A u,
$$



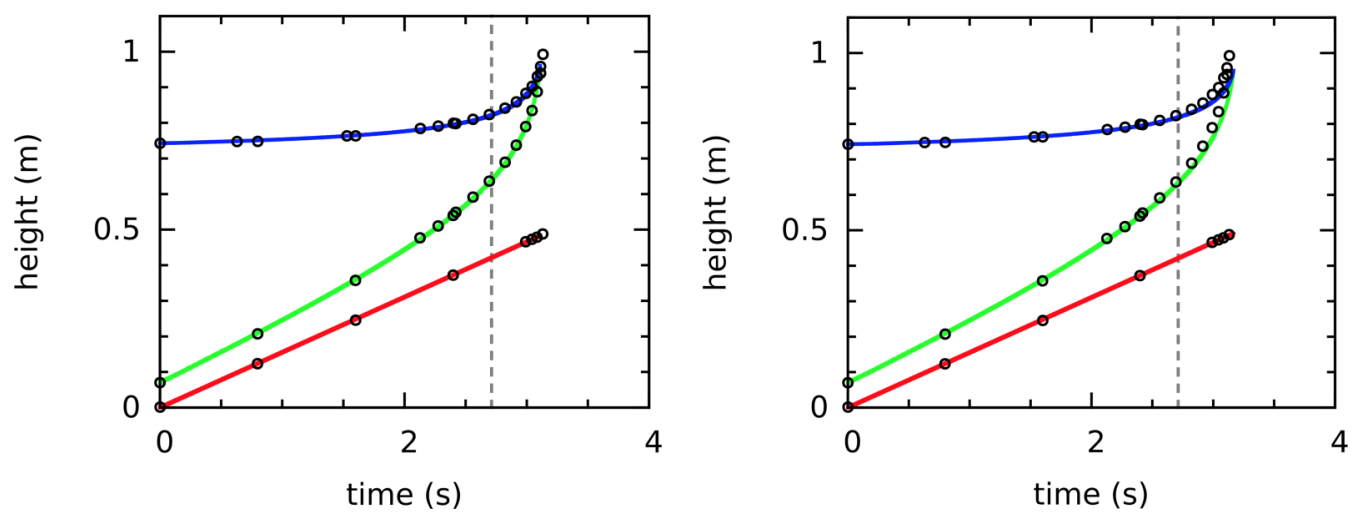

FIG. 4. Comparison of the present model (left) and the model of Ref. [10] for case (b) of Ref. [11]. The lines are, in ascending order, the positions of the bubble base, of the bubble top and of the free surface of the liquid, all vs. time. The circles are the experimental data from Ref. [11]. In the experiment the Galilei number was 91.3 , the Morton number 0.08, and the Eötvös number 179. The vertical dashed line marks the time $t_{c}$ estimated from Eq. (14) at which the critical bubble length Eq. (13) is reached.

from which, after using Eq. (4),

$$
A u=A_{b} \dot{\ell}
$$

With this relation the momentum Eq. (31) becomes

$$
\eta \rho h \ddot{\ell}=p_{g}-\left(p_{t}+\rho g h\right)-4 \frac{h}{d} \tau_{w},
$$

with $\eta=A_{b} / A$ as before.

Reference [10] presents a momentum equation for the column of liquid above the bubble which is written with reference to the center of mass of the column without a first-principles derivation. With the present notation, this equation [Eq. (6) of the reference] can be written as

$$
\frac{1}{2}(1+\eta) \rho h \ddot{\ell}=p_{g}-\left(p_{t}+\rho g h\right)-4 \frac{h}{\eta d} \tau_{w},
$$

This equation reduces to our form Eq. (35) if $\eta \simeq 1$, i.e., $A_{b} \simeq A$. According to Ref. [18], the minimum thickness of the liquid film around a Taylor bubble is found in the high-Reynolds-number range and is somewhat less than $10 \%$ of the tube radius, which translates into $1-\eta \sim 0.2$. While small, this value may not be completely negligible.

We have found that comparison with experiment improves by a few percent if, instead of taking the top bubble surface to be flat, we take to be a hemisphere with the same radius $r$ as the cylindrical body. In this case, in place of Eq. (1), the gas pressure is calculated from

$$
p_{g}\left(\ell-\frac{1}{3} r\right)=p_{g 0}\left(\ell_{0}-\frac{1}{3} r\right) \text {. }
$$

Figure 4 shows a comparison of Eq. (35), with $p_{g}$ calculated from Eqs. (37) and (36), with one of the four sets of data in Fig. 2 of Ref. [10]. The comparison is very similar for the three other cases that we have tested. In the experiment the tube continued above the initial level of the free surface as in the right panel of Fig. 3. The liquid used had a viscosity of $0.124 \mathrm{~Pa}$ s, a density of $862 \mathrm{~kg} / \mathrm{m}^{3}$ and a surface tension of $0.032 \mathrm{~N} / \mathrm{m}$; the tube diameter was $26 \mathrm{~mm}$. The pressure $p_{t}$ on the liquid free surface could be varied and the comparison that we present concerns the experiments with $p_{t}=77 \mathrm{~Pa}$ (case $b$ in Fig. 2 of the reference); the data have been deduced from the figure in the original publication with the aid of suitable software. These low-pressure data are particularly 
interesting because, as mentioned in Sec. II, in this case the expansion is violent and dynamic effects are particularly significant. Since the initial conditions for the experiment are given only approximately in the reference, we deduced them from the data points at the earliest time shown in the original figure. We fitted by a straight line (disregarding the last cluster of closely spaced points which could not be estimated very accurately) the data for the position of the bubble base vs. time to deduce $u_{B}$, which is an input for both models. We found $u_{B}=0.1553 \mathrm{~m} / \mathrm{s}$, less than the $0.177 \mathrm{~m} / \mathrm{s}$ given by the standard correlation $u_{B}=0.351 \sqrt{g d}$ (see, e.g., Refs. [16,19]). This difference reflects the fairly strong viscous effects in the conditions of the experiment signaled by the value of the Galilei number,

$$
\mathrm{Ga}=\frac{d \sqrt{g d}}{v},
$$

which equals 91.3; the Morton number is Mo $=0.08$ and the Eötvös number Eo $=179$. In this and the following figures the vertical dashed line marks the time $t_{c}$ estimated from Eq. (14) at which the critical lengths Eq. (7) or Eq. (13) are reached. It is seen that, at approximately this time, the rate of expansion of the bubble starts to dramatically increase.

Both models give an acceptable performance for the prediction of the flow evolution in the tube. It is impossible to decide between them solely on the basis of this and similar comparisons as we have found that the precise results are very sensitive to the initial conditions, the value of $u_{B}$ and other aspects of the model such as the thickness of the film. As a matter of fact, the curves in Fig. 2 of Ref. [11] appear slightly different from our reconstruction on the basis of Eq. (36) probably due to the use of somewhat different initial conditions.

\section{A. Energy balance}

It is interesting to consider the energy balance implicit in Eq. (30). Upon multiplying this equation by $u$ we may write

$$
\frac{1}{2} A h \rho \frac{d u^{2}}{d t}=A p_{g} u-A p_{t} u-\rho g A h u-\pi d h \tau_{w} u,
$$

or, by Eq. (34),

$$
d\left(\frac{1}{2} A h \rho u^{2}\right)=A_{b} p_{g} d \ell-A p_{t} u d t-\rho g A h u d t-\pi d h \tau_{w} u d t+\frac{1}{2} A \rho u^{2} d h .
$$

The first two terms in the right-hand side are the net work $d W_{p}=A_{b} p_{g} d \ell-A p_{t} u d t$ performed by the pressure forces; the next term is the potential energy gain $d E_{p}=\rho g A h u d t$; the next-to-last term is the energy lost to viscous and turbulent dissipation, $d E_{\mu}=\pi d h \tau_{w} u d t$, and the left-hand side is the change in the kinetic energy of the liquid in the tube:

$$
d K=d\left(\frac{1}{2} A h \rho u^{2}\right) .
$$

The previous relation can therefore be rearranged to give

$$
d W_{p}=d K+d E_{p}+d E_{\mu}-\frac{1}{2} A \rho u^{2} d h .
$$

The first three terms in the right-hand side are as expected, but it is interesting to look deeper into the last term. For a tube that does not continue above the initial liquid level, from Eqs. (4) and (20) with $\alpha=1$, we can write

$$
-\frac{1}{2} A \rho u^{2} d h=\frac{1}{2} A \rho u^{2}\left(d \ell+u_{B} d t\right)=\frac{1}{2} A \rho u^{2}(u d t)+\frac{1}{2} \rho u^{2}\left[A u_{B} d t+\left(A-A_{b}\right) d \ell\right],
$$

where use has been made of Eq. (34). The first term is the kinetic energy ejected from the tube. The remaining terms account for the kinetic energy lost due to the fact that some liquid must come to rest filling the space under the rising bubble and that some liquid must come to rest around the bubble top as it advances by an amount $d \ell$. 


\section{B. Scaling}

A quantity suitable to characterize the damage potential of the Taylor bubble is the total kinetic energy $K_{\text {ej }}$ ejected from the tube between the initial and final times. On the basis of the previous argument this is given by

$$
K_{\mathrm{ej}}=\int_{0}^{t_{f}} \frac{1}{2} \rho A u^{3} d t .
$$

To enhance their value, in the presentation of numerical results it is useful to adopt dimensionless variables. To this end we use $h_{t}=p_{t} / \rho g$ as fundamental length and $\sqrt{p_{t} / \rho g^{2}}$ as fundamental time writing, for example,

$$
\hat{\ell}=\frac{\ell}{p_{t} / \rho g}, \quad \hat{t}=\frac{t}{\sqrt{p_{t} / \rho g^{2}}} .
$$

As a consequence, velocities are nondimensionalized by $\sqrt{p_{t} / \rho}$. In terms of these quantities the dynamical Eq. (35) becomes

$$
\eta \hat{h} \frac{d^{2} \hat{\ell}}{d \hat{t}^{2}}=\frac{\Gamma}{\hat{\ell}}-(1+\hat{h})-2 \eta^{2} \Pi \hat{h}\left(\frac{d \hat{\ell}}{d \hat{t}}\right)^{2} f .
$$

The parameter $\Gamma=\left(1+\hat{h}_{0}\right) \hat{\ell}_{0}$ was defined earlier in Eq. (11) and is, in a sense, related to the initial energy of the bubble. The new parameter,

$$
\Pi=\frac{p_{t}}{\rho g d},
$$

compares the hydrostatic head at the top of the tube to the tube diameter. The friction factor is a function of the Reynolds number which may be written

$$
\mathrm{Re}=\frac{u d}{v}=\eta \mathrm{Ga} \sqrt{\Pi} \frac{d \hat{\ell}}{d \hat{t}},
$$

with Ga the Galilei number defined in Eq. (38). Other quantities entering the theory are the velocity of the bubble bottom, which becomes $\hat{u}_{B}=\operatorname{Fr}(\mathrm{Ga}) / \sqrt{\Pi}$, with $\mathrm{Fr}$ the Froude number which is expressed in terms Ga (see, e.g., Ref. [20]) and $\eta$ which can also be expressed in terms of Ga (see, e.g., Ref. [18]). Thus, the results will depend on the parameters $\Pi$ and $\mathrm{Ga}$ in addition to the initial conditions $\hat{\ell}_{0}$ and $\hat{h}_{0}$. While $\Gamma$ can be written in terms of these initial conditions, it will be seen from the numerical results that the combination of these quantities embodied by $\Gamma$ plays an important role. It may be noted that surface tension (which we neglect on account of the smallness of its effects in the vast majority of applications of our study), would introduce an additional dimensionless parameter.

For typical applications to volcanos relevant ranges for the liquid density and viscosity are $1300<\rho<2600 \mathrm{~kg} / \mathrm{m}^{3}$ and $1<\mu<10^{3} \mathrm{~Pa} \mathrm{~s}$ and for the conduit diameter $3<d<6 \mathrm{~m} \mathrm{[8,9]}$. The corresponding range for the dimensionless parameters $\Pi$ and Ga are, approximately, $0.6<$ $\Pi<2.5$ and $1<\mathrm{Ga}<50,000$. For typical applications in the oil industry relevant ranges for the liquid density and viscosity are $800<\rho<1000 \mathrm{~kg} / \mathrm{m}^{3}$ and $10^{-3}<\mu<10^{-1} \mathrm{~Pa} \mathrm{~s}$ and for the conduit diameter $0.1<d<0.3 \mathrm{~m}[21,22]$. The corresponding range for the dimensionless parameters $\Pi$ and $\mathrm{Ga}$ are, approximately, $30<\Pi<120$ and $4000<\mathrm{Ga}<10^{6}$.

We re-write the energy balance Eq. (42) in dimensionless form as

$$
\frac{d \hat{W}_{p}}{d \hat{t}}=\frac{d \hat{E}_{k}}{d \hat{t}}+\frac{d \hat{E}_{p}}{d \hat{t}}+\frac{d \hat{E}_{\mu}}{d \hat{t}},
$$




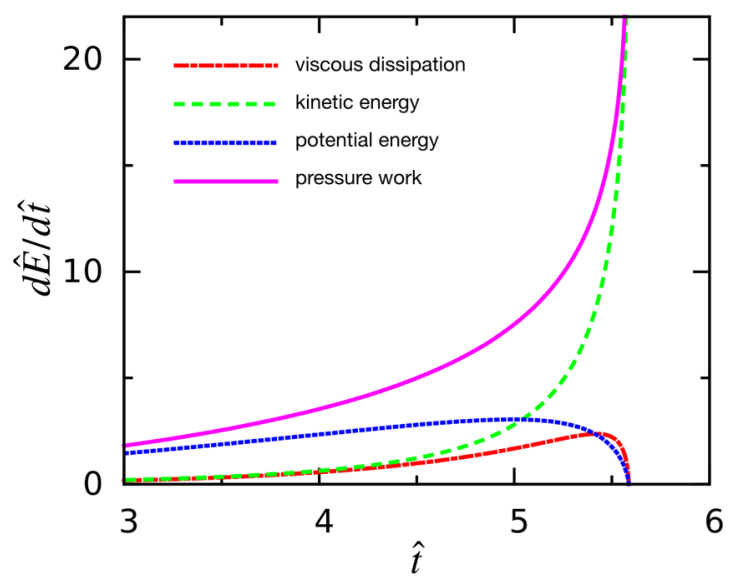

FIG. 5. Time dependence of the three terms in the energy balance Eq. (49) with $\Pi=1, \mathrm{Ga}=50, \hat{h}_{0}=5$, $\hat{\ell}_{0}=16.7$, and $\Gamma=100$. The top line (continuous, purple) is the rate of work done by the pressure forces (left-hand side of the equation). The other lines are the rate of change of the kinetic energy of the liquid in the tube (long dashes, green), the rate of change of the potential energy (dotted, blue) and the rate of energy dissipation (dash-dots, red). The sum of the three lower lines equal the rate of pressure work within numerical error.

in which we have set

$$
\frac{d \hat{E}_{k}}{d \hat{t}}=\frac{d \hat{K}}{d \hat{t}}-\hat{u}^{2} \frac{d \hat{h}}{d \hat{t}}
$$

This quantity is the rate of change of the kinetic energy in the tube not including the change in the height of the liquid column. According to the definitions Eq. (45), here all quantities with dimension energy have been normalized by $\frac{1}{2} A p_{t}^{2} / \rho g$.

Figure 5 shows the time dependence of the terms in Eq. (49) for a typical case with $\Pi=1$, $\mathrm{Ga}=50, \hat{h}_{0}=5, \hat{\ell}_{0}=16.7$, and $\Gamma=100$. The potential energy and the energy dissipated first increase with time as the liquid column accelerates but quickly decrease to zero as the mass of liquid in the tube becomes smaller and smaller. In the final stages of the process most of the pressure work goes into the kinetic energy of the liquid in the tube and is ultimately responsible for the violence of the phenomenon.

The dependence of the kinetic energy $\hat{K}_{e j}$ ejected from the tube mouth on the parameters of the problem is shown in Figs. 6 and 7. The two panels in Fig. 6 show the dependence of $\hat{K}_{\text {ej }}$ on the initial bubble depth $\hat{h}_{0}$ and the ratio of the initial depth to the initial length, $\hat{h}_{0} / \hat{\ell}_{0}$, in both cases for constant values of $\Gamma=\left(1+\hat{h}_{0}\right) \hat{\ell}_{0}$ keeping $\Pi=1$ and $\mathrm{Ga}=50$ fixed. In both cases $\hat{K}_{\mathrm{ej}}$ at first increases as $\hat{h}_{0}$ or $\hat{h}_{0} / \hat{\ell}_{0}$ increase but then saturates at a constant value which is an increasing function of $\Gamma$. Increasing $\hat{h}_{0}$ or $\hat{h}_{0} / \hat{\ell}_{0}$ with $\Gamma$ constant has the effect of decreasing $\hat{\ell}_{0}$, which can be seen as an increase of the initial compression of the bubble. Transition to constancy very nearly coincides with $\hat{h}_{0} / \hat{\ell}_{0}=1$, marked by a dashed line in the right panel. Thus, once $\hat{h}_{0}$ becomes larger than $\hat{\ell}_{0}$, the only thing that matters is the initial bubble enthalpy as approximately measured by $\Gamma$.

Another perspective on the ejected kinetic energy is given in Fig. 7, where $\hat{K}_{\text {ej }}$ is seen to have a strikingly close proportionality to $\Gamma$. The slope of the lines decreases with the Galilei number as a consequence of the increasing amount of dissipation; one observes also a very slight downward curvature as $\mathrm{Ga}$ decreases. Increasing $\Pi$, i.e., in particular, increasing the pressure $p_{t}$ at the tube top, decreases the ejected kinetic energy by reducing the expansion rate of the bubble. 

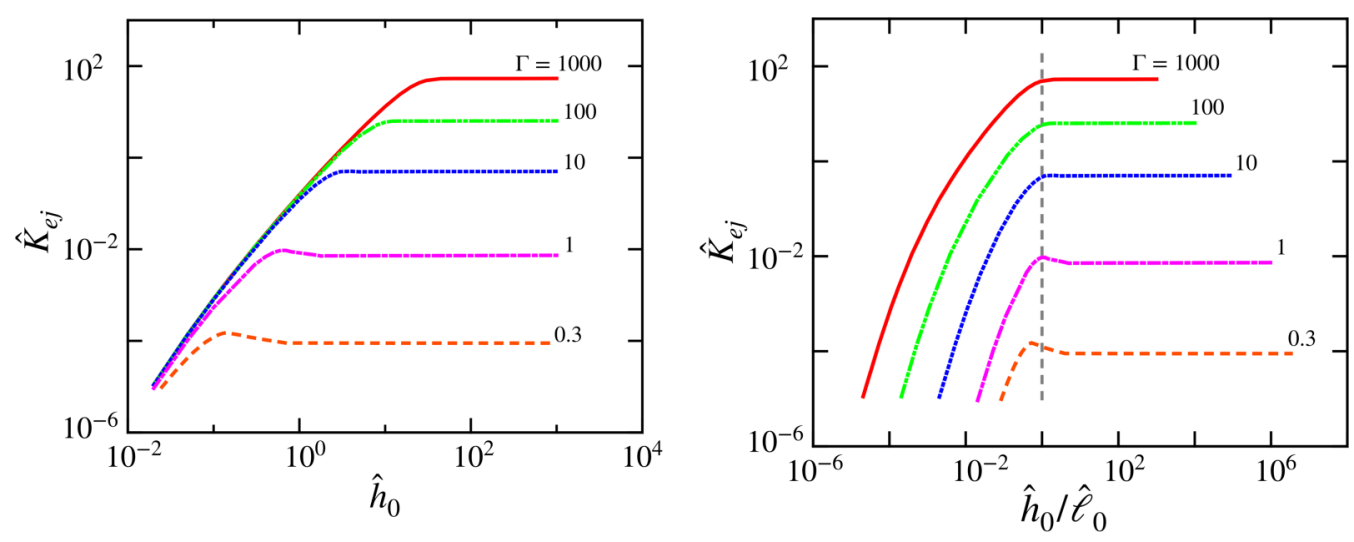

FIG. 6. Total kinetic energy ejected from the tube in dependence of the dimensionless initial bubble depth $\hat{h}_{0}$ (left) and of the ratio $\hat{h}_{0} / \hat{\ell}_{0}$ of the initial depth to the initial bubble length, all with $\Pi=1$ and $\mathrm{Ga}=50$ fixed. In ascending order, the lines correspond to increasing values of the parameter $\Gamma$, an approximate measure of the initial bubble enthalpy.

\section{NAVIER-STOKES SIMULATION}

To gain more confidence on the results obtained from the simplified models of the previous two sections, we have carried out a full Navier-Stokes simulation with the open-source code OpenFOAM version 5 .

The code uses a finite-volume framework, with a second-order backward discretization for the time-derivative terms and a second-order linear upwind scheme for convection terms. A volume of fluid (VOF) method for two-phase flow is used to track the motion of interfaces. The void fraction is solved with a van Leer limiter and time stepping is effected with the Crank-Nicolson scheme. To reduce the computational time, a 5-degree wedge is simulated assuming axial symmetry. We used 25 mesh cells in the radial direction as in Ref. [23], which results in about 8 cells in the film region of the bubble; a grid convergence analysis proved this resolution to be sufficient.

Figure 8 shows a sequence which illustrates the rapid expansion of the bubble as it approaches the free surface. Separating successive images by the same time interval gives a clear impression

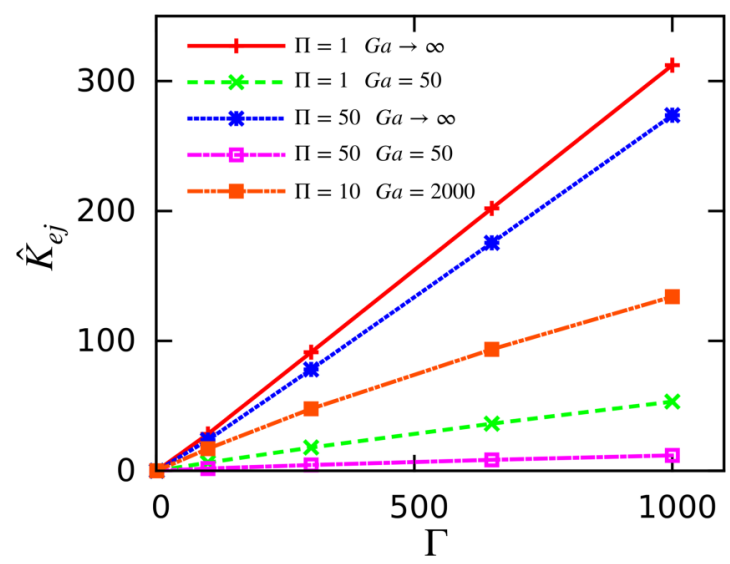

FIG. 7. Total kinetic energy ejected from the tube in dependence of the parameter $\Gamma$, an approximate measure of the initial bubble enthalpy, for various values of $\Pi$ defined in Eq. (47) and of the Galilei number Ga. 


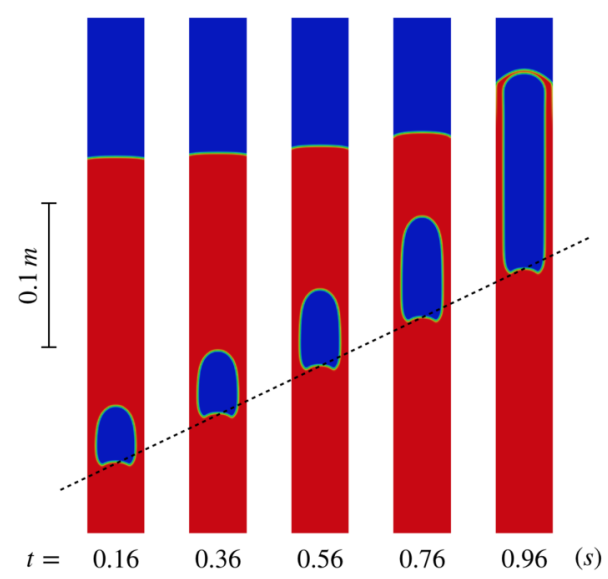

FIG. 8. A sequence of snapshots equally spaced in time showing the rapid expansion of the bubble as it approaches the free surface as calculated with OpenFOAM. The Galilei number is 51, the Morton number 0.6 and the Eötvös number 160. Note the constant rise velocity of the bubble base.

of the rapidity of the phenomenon and of the constant rise velocity of the bubble base. The fluid used in this example has a viscosity of $0.5 \mathrm{~Pa} \mathrm{~s}$, a density of $1000 \mathrm{~kg} / \mathrm{m}^{3}$ and a surface tension of $0.1 \mathrm{~N} / \mathrm{m}$; the tube diameter is $40 \mathrm{~mm}$ and the pressure on the liquid free surface a the top of the tube is $200 \mathrm{~Pa}$. The values of the dimensionless parameters are $\mathrm{Ga}=51, \mathrm{Mo}=0.6$, and Eo $=160$. The initial distance of the bubble tip from the free surface is $0.2 \mathrm{~m}$. The calculation was initiated giving the bubble the form of cylinder with a height of $14 \mathrm{~mm}$ and a diameter of $26 \mathrm{~mm}$, capped by hemispheres at both ends. This initial shape changes to the one shown in the first image already in the first few time steps. The calculated rise velocity of the bubble base was $0.168 \mathrm{~m} / \mathrm{s}$, significantly smaller than the value $0.222 \mathrm{~m} / \mathrm{s}$ predicted by the standard correlation $0.351 \sqrt{d g}$, but close to the value $0.163 \mathrm{~m} / \mathrm{s}$ from the generalized correlation of Ref. [20] which includes viscous effects.

A comparison of the OpenFOAM results with the experimental data for the same case of the previous Fig. 4 (case $b$ in Fig. 2 of Ref. [11]) is shown in Fig. 9, where the open circles are the

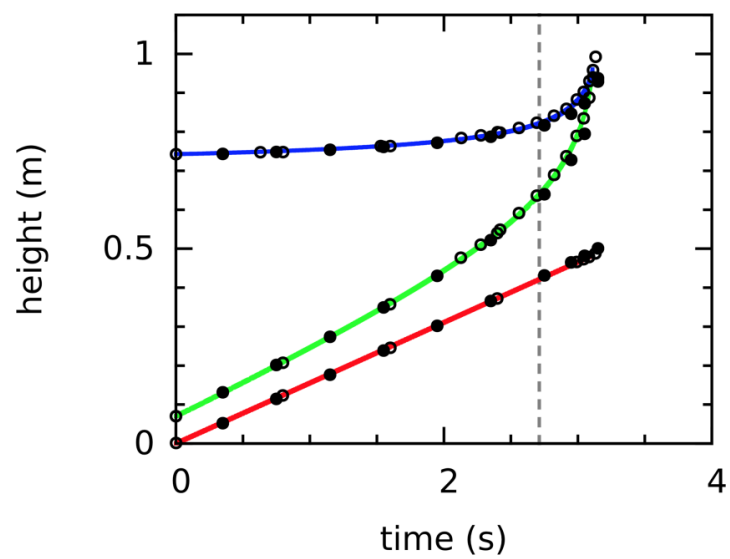

FIG. 9. Comparison of the dynamic model of Sec. III (lines) with the OpenFOAM results (black circles) and the experimental data for the same case of Ref. [11] shown in Fig. 4. The lines are, in ascending order, the positions of the bubble base, of the bubble top and of the free surface of the liquid. The vertical dashed line marks the time $t_{c}$ estimated from Eq. (14) at which the critical bubble length Eq. (13) is reached. 
experimental data, the black circles the OpenFOAM results and the lines the results of the dynamic model of the previous section. The velocity of the bubble base computed with OpenFOAM is about $0.157 \mathrm{~m} / \mathrm{s}$, to be compared with $0.1553 \mathrm{~m} / \mathrm{s}$ obtained by a linear fit to the data.

Simulations of this type are clearly preferable to the use of approximate models, but they incur two serious difficulties. In the first place, demonstrating the effect with realistic dimensions and property values incurs a considerable computational burden. Using for example the simple estimate of Eq. (11) for critical conditions, we see that, with an initial bubble length of $1 \mathrm{~m}$, with atmospheric pressure at the top of the tube so that $h_{t}=p_{t} / \rho g \simeq 10 \mathrm{~m}$, the initial bubble depth should be more than $100 \mathrm{~m}$ for the violent expansion to occur. The number of cells necessary for such a simulation would be quite substantial. Second, the small surface tension effect in realistic conditions causes capillary waves along the film and other instabilities which require a very fine mesh and a correspondingly small time step.

\section{DRIFT FLUX MODEL-FIXED FRAME}

The dynamic model is fast and simple, but its derivation is based on several simplifying assumptions the general validity of which cannot be guaranteed in all cases. The axisymmetric Navier-Stokes simulation of the previous section is more reliable, but it incurs a considerable computational burden, particularly in the case of the very long tubes encountered in deep-water drilling. An attractive compromise between accuracy and cost is the drift-flux model. We follow the formulation of Ref. [24].

The model consists of the continuity equations for the two phases, one for the gas,

$$
\frac{\partial\left(\alpha_{G} \rho_{G}\right)}{\partial t}+\frac{\partial\left(\alpha_{G} \rho_{G} u_{G}\right)}{\partial z}=0
$$

and one for the liquid, assumed incompressible,

$$
\frac{\partial \alpha_{L}}{\partial t}+\frac{\partial\left(\alpha_{L} u_{L}\right)}{\partial z}=0
$$

where the subscripts $G$ and $L$ denote the gas and liquid phases, respectively and $\alpha$ denotes the volume fraction with $\alpha_{G}+\alpha_{L}=1$. In the model only one momentum equation is solved,

$$
\frac{\partial(\rho u)}{\partial t}+\frac{\partial\left(\rho u^{2}\right)}{\partial z}=-\frac{\partial p}{\partial z}+\mu \frac{\partial^{2} u}{\partial z^{2}}-\frac{\partial Q}{\partial z}-\rho g+\left(\frac{\partial p}{\partial z}\right)_{f}
$$

where the mixture density $\rho$ and center-of-mass velocity $u$ are

$$
\rho=\alpha_{L} \rho_{L}+\alpha_{G} \rho_{G}, \quad \rho u=\alpha_{L} \rho_{L} u_{L}+\alpha_{G} \rho_{G} u_{G} .
$$

The term $Q$ arises from the nonlinearity of the momentum flux and is given by

$$
Q=\frac{\alpha_{G} \rho_{G} \rho_{L}}{\alpha_{L} \rho} V^{2},
$$

with $V$ the drift velocity given by

$$
V=0.37 \sqrt{\frac{\left[\left(\rho_{L}-\rho_{G}\right) \alpha_{L} g-(\partial p / \partial z)_{f}\right] d}{\alpha_{L} \rho_{L}}} .
$$

The velocities of the individual phases can be expressed in terms of $u$ and $V$ by

$$
u_{L}=u-\frac{\alpha_{G} \rho_{G}}{\alpha_{L} \rho} V, \quad u_{G}=u+\frac{\rho_{L}}{\rho} V .
$$


The term $(\partial p / \partial z)_{f}$ in Eq. (53) is the pressure gradient caused by dissipative mechanisms and is calculated from

$$
\left(\frac{\partial p}{\partial z}\right)_{f}=-\frac{2}{d} f \rho_{L} u_{L}\left|u_{L}\right|
$$

Expressions for the friction factor $f$ were given earlier in Eqs. (26) and (27) above. In using these relations the Reynolds number is based on the hydraulic diameter of the liquid at each cross section. Finally, an equation of state for gas is needed to close the system, which we take of the isothermal form

$$
\rho_{G}=\frac{p}{c^{2}},
$$

with $c$ the isothermal speed of sound in the gas. In the numerical implementation of the model it is convenient to rewrite the gas continuity Eq. (51) in the following form, with only one unknown differentiated with respect to time

$$
\alpha_{G} \frac{\partial \rho_{G}}{\partial t}+\rho_{G} \frac{\partial\left(\alpha_{L} u_{L}\right)}{\partial z}+\frac{\partial\left(\alpha_{G} \rho_{G} u_{G}\right)}{\partial z}=0 ;
$$

this equation is found by using Eq. (52) to eliminate $\partial \alpha_{G} / \partial t$.

Both the gas volume fraction and the liquid velocity are set to zero at the bottom of the tube. At the top of the tube the velocity gradient is set to zero and the pressure is kept constant to simulate the case in which the liquid surface is at the mouth of the tube as sketched in Figs. 1 and 3, left.

The equations are discretized in a finite volume manner with first-order upwinding. The mesh used is uniform and all the variables are colocated at cell centers.

Initialization of the drift flux model requires some care as the presence of the prescribed drift velocity $V$ Eq. (56) prevents the gradual build-up of the velocity and pressure fields in the system. Ideally, the simulation should be started with conditions that are already fully compatible with the model. Since these conditions are unknown, we proceed as follows. Initially the bubble is taken to be a cylinder surmounted by a hemispherical cap, the fluid velocity is assumed to vanish and the pressure is set to an arbitrary constant. We then solve the momentum Eq. (53) and gas-density Eq. (60) for the initially prescribed volume fraction distribution; since the volume fraction is kept fixed, its evolution Eq. (52) is not used. The process is repeated until velocity and pressure have converged to constant values. This signals the fact that the pressure and velocity fields have become compatible with the assumed volume fraction distribution and the integration of the complete model can begin.

\section{DRIFT FLUX MODEL-MOVING FRAME}

Although the execution time for the drift-flux model is much shorter than for axisymmetric Navier-Stokes OpenFOAM simulation, it still becomes unacceptably burdensome in the case of a very long tube. The structure of the single-phase regions of the flow, however, suggests how to achieve a major simplification.

Away from the two-phase region, $\alpha_{G}=0$ so that $\rho=\rho_{L}$ and $u=u_{L}$. From the liquid continuity equation we then deduce that

$$
u_{L}=u_{L}(t)
$$

The momentum equation becomes, therefore,

$$
\frac{\partial\left(\rho_{L} u_{L}\right)}{\partial t}=-\frac{\partial p}{\partial z}-\rho_{L} g+\left(\frac{\partial p}{\partial z}\right)_{f} .
$$

Since the left-hand side and the frictional pressure drops are only functions of time, this relation implies that the pressure gradient is independent of position in the single-phase liquid region. With 


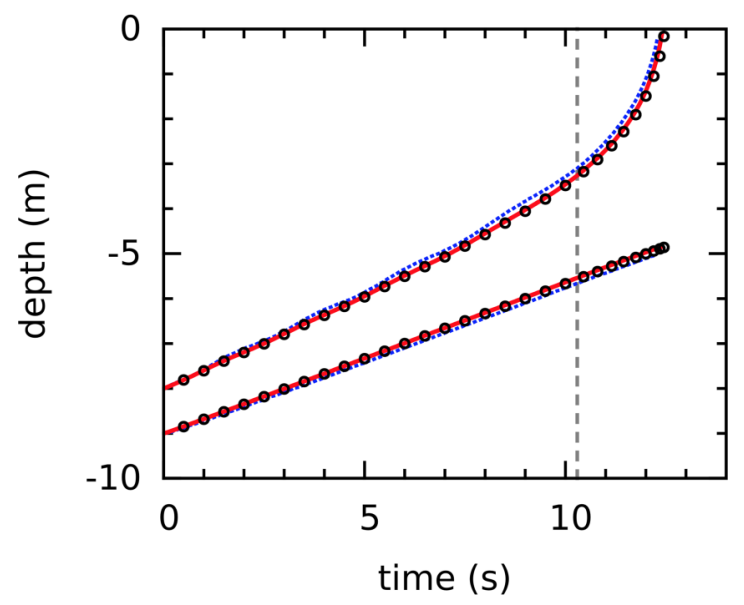

FIG. 10. Comparison of the drift flux model as implemented with a fixed frame (lines) and with a moving frame (circles). The tube height is $8 \mathrm{~m}$ and its diameter $0.1 \mathrm{~m}$; the Galilei number has the value of $5.8 \times 10^{4}$. The dashed line has been computed with 1000 cells and the solid line with 8000; the moving frame calculation has been carried out with 1000 cells. The lower and upper sets of lines mark the position of the bubble base and top, respectively. The vertical dashed line marks the time $t_{c}$ estimated from Eq. (14) at which the critical bubble length Eq. (7) is reached.

this remark we are able to focus only on the relatively short section of tube containing the bubble and the flow region affected by its motion, so that it is possible to adopt a grid sufficiently fine to have a good resolution independently of the tube length. For this purpose, we adopt a reference system moving upward with the same velocity as the bottom of the bubble. The time-dependent pressure gradient is determined by requiring that the pressure gradient at the top of the moving domain be such that, when multiplied by the height of the liquid column above it, it reproduces the prescribed pressure $p_{t}$ acting on the free surface. The remaining boundary conditions are imposed at the bottom of the moving domain requiring that the liquid leave the domain at the rate determined by the rise velocity of the latter and that the gas volume fraction vanish.

Figure 10 compares the results obtained with a fixed and moving frame for a bubble initially $1 \mathrm{~m}$ long under an 8-m-long water column in a tube with a diameter of $0.1 \mathrm{~m}$. The pressure on the liquid free surface is $1000 \mathrm{~Pa}$. The Galilei number has the value of $5.8 \times 10^{4}$. It is found that the dynamics of the bubble has some dependence on the film thickness with which it is initialized. Here we used Eq. (4.2) from the recent work of [18] to initialize the film to a thickness of $4.10 \mathrm{~mm}$; the corresponding liquid volume fraction is $\alpha_{L}=0.157$. The dashed and solid lines are the drift-flux results as computed for a fixed frame with 1000 and 8000 cells. The circles are the results for the moving frame obtained with 1000 cells; initially the calculation focuses on a 2-m-long section of tube centered on the bubble, which is gradually increased as the bubble expands. The latter results are virtually indistinguishable from the fixed-frame ones obtained with a significantly larger number of cells. Since the situation simulated is one in which the initial liquid free surface is at the top of the tube so that the liquid spills out maintaining the same level, it is not necessary to show the position of the liquid free surface as in the previous Figs. 4 and 9.

Figure 11 shows a comparison of the moving-frame drift-flux model (solid lines) and the dynamic model of Sec. III for the same case as the previous figure. The upper and lower lines show the position of the top and bottom of the bubble versus time. The rise velocity as calculated from the drift-flux model is $0.334 \mathrm{~m} / \mathrm{s}$, to be compared with the value $0.337 \mathrm{~m} / \mathrm{s}$ given by the correlation of Ref. [20].

The two set of results are very close over most of the history of the bubble rise, but some differences are encountered in the final strongly dynamic phase. We hypothesize that the discrepancy 


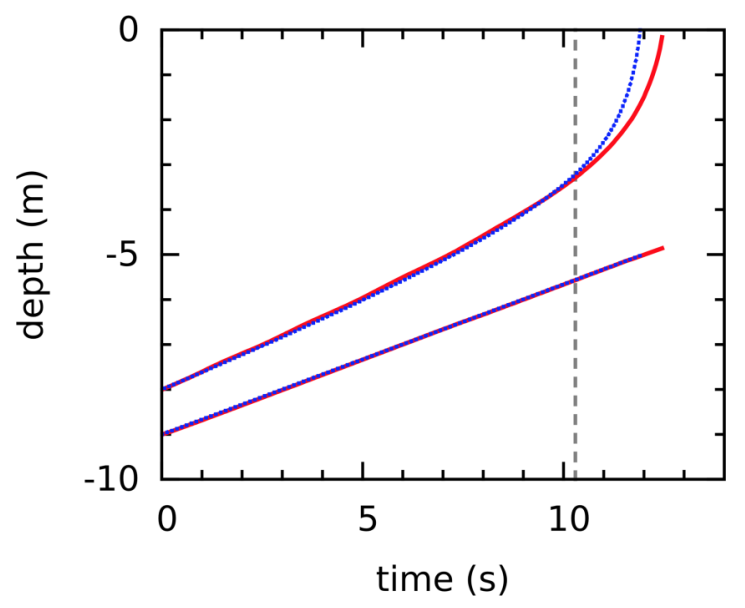

FIG. 11. Comparison of the drift-flux model (solid lines, red) with the dynamic model of Sec. III for a situation in which the liquid spills out of the top of the tube for the same case as in the previous figure. The lower and upper pairs of lines mark the position of the bubble base and top, respectively. The vertical dashed line marks the time $t_{c}$ estimated from Eq. (14) at which the critical bubble length Eq. (7) is reached.

arises from an incorrect evaluation of the liquid film thickness around the bubble for which the drift flux model does not contain the correct physics due to its one-dimensional nature. In spite of this relatively minor difference, it can be said that the two models make predictions in substantial agreement with each other.

The use of a moving frame is particularly attractive in the case of a very long tube. As our concluding example we show the rise of a gas bubble in a water-filled $1000 \mathrm{~m}$ long tube with a diameter of $0.1 \mathrm{~m}$. The initial bubble diameter is $0.0918 \mathrm{~m}$ and the initial bubble length $1 \mathrm{~m}$. Figure 12 shows the position of the bottom and top of the bubble and Fig. 13 the liquid velocity as it spills out of the top of the tube, both figures focusing on the last stages of the bubble rise and expansion; as in the previous figures, the vertical dashed line shows the time $t_{c}$ estimated from Eq. (14) at which the critical bubble length Eq. (7) is reached. The second figure is particularly

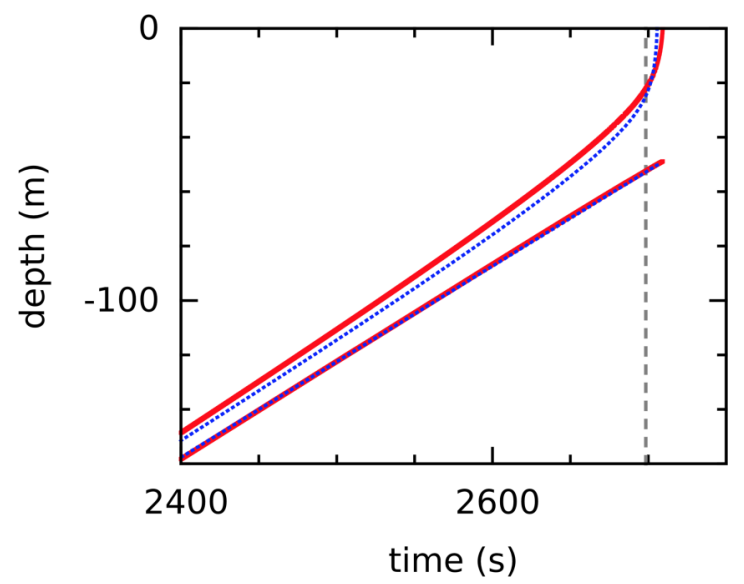

FIG. 12. Comparison of the drift-flux model (solid lines, red) with the dynamic model of Sec. III in the final stage of the bubble rise and expansion. In the situation simulated the liquid spills out of the top of the tube. The tube height is $1000 \mathrm{~m}$ and its diameter $0.1 \mathrm{~m}$; the Galilei number has the value of $5.8 \times 10^{4}$. The vertical dashed line marks the time $t_{c}$ estimated from Eq. (14) at which the critical bubble length Eq. (7) is reached. 


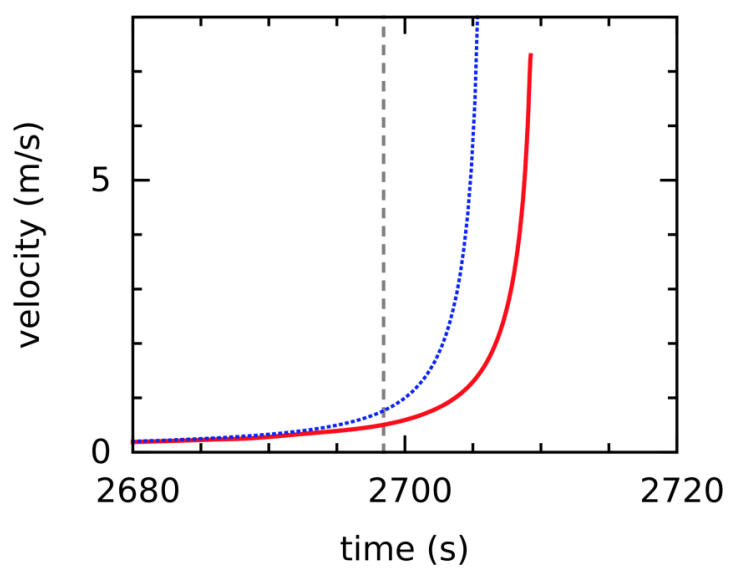

FIG. 13. Liquid velocity as it spills out of the tube top in the final stages of the bubble rise and expansion for the case of the previous figure. It will be appreciated that the velocity remains well below $0.1 \mathrm{~m} / \mathrm{s}$ for about $45 \mathrm{~min}$ and rises to nearly $10 \mathrm{~m} / \mathrm{s}$ in the last $3 \mathrm{~s}$ or so.

striking: the velocity of the spilling liquid remains of the order of a few $\mathrm{cm} / \mathrm{s}$ for about 45 minutes and shoots up to nearly $10 \mathrm{~m} / \mathrm{s}$ in the last few seconds leaving virtually no time to adopt mitigating measures if this was oil spilling out of a riser.

\section{SUMMARY AND CONCLUSIONS}

In this paper we have used several models to study the rise and violent expansion of a large gas bubble in a vertical tube. We have shown that the rapid expansion phase can be reached in very long tubes or when the pressure at the tube top is substantially below atmospheric. The phenomenon is of interest in volcanology, where it is thought to be responsible for eruptions of the Strombolian type, which intermittently eject material to heights up to hundreds of meters. In the oil industry it can give rise to major accidents as the onset of the rapid bubble expansion can be very sudden not allowing sufficient time for the adoption of adequate counter-measures (Fig. 13). The early detection of the presence of large volumes of free gas in risers becomes then an issue of paramount concern. If gas is detected early, it can be allowed to rise using the diverter to handle the low-velocity oil expelled from the tube top before the rapid expansion sets in. When the bubble has risen sufficiently, the tube can be sealed until the bubble rises to the top. This process causes the pressure to rise, but this rise is much smaller and, therefore, much less dangerous than what it would be if the tube was sealed when the bubble was at depth.

\section{ACKNOWLEDGMENTS}

The authors are grateful to Dr. Amit Amritkar (Department of Mechanical Engineering, University of Houston) for his contribution to the early stages of this project and to Taher Chegini for his help on the use of OpenFOAM. Research reported in this paper was supported by the Gulf Research Program of the National Academies of Sciences, Engineering, and Medicine under Award No. GRP2000008864. The content is solely the responsibility of the authors and does not necessarily represent the official views of the Gulf Research Program or the National Academies of Sciences, Engineering, and Medicine.

[1] Drilling rig explosion and fire at the Macondo well. Investigation Report 2010-10-I-OS. U.S. Chemical Safety and Hazard Investigation Board (2016). 
[2] C. S. Avelar, P. R. Ribeiro, and K. Sepehrnoori, Deepwater gas kick simulation, J. Petr. Sci. Eng. 67, 13 (2009).

[3] H. V. Nickens, A dynamic computer model of a kicking well, SPE Drill. Engineer. 2, 159 (1987).

[4] N. Velmurugan, J.-M. Godhavn, and E. Hauge, Dynamic simulation of gas migration in marine risers, in Society of Petroleum Engineers (SPE-180022-MS) (SPE, 2016).

[5] J. Xie, X. Zhang, Y. Tang, W. Yan, Q. Shao, and Y. Bo, Transient simulation of the blowing-out process of the air pockets in vertical wellbore, Appl. Thermal Eng. 72, 97 (2014).

[6] L. M. T. Santos, M. T. M. Sena Esteves, and M. N. Coelho Piñeiro, Effect of gas expansion on the velocity of individual Taylor bubbles rising in vertical columns with water: Experimental studies at atmospheric pressure and under vacuum, Chem. Eng. Sci. 63, 4464 (2008).

[7] R. G. Sousa, A. M. F. R. Pinto, and J. B. L. M. Campos, Effect of gas expansion on the velocity of a Taylor bubble: PIV measurements, Int. J. Multiphase Flow 32, 1182 (2006).

[8] E. del Bello, E. W. Llewellin, J. Taddeucci, P. Scarlato, and S. J. Lane, An analytical model for gas overpressure in slug-driven explosions: Insights into strombolian volcanic eruptions, J. Geophys. Res. 117, B02206 (2012).

[9] H. M. Gonnermann and M. Manga, The fluid mechanics inside a volcano, Annu. Rev. Fluid Mech. 39, 321 (2007).

[10] M. R. James, S. J. Lane, and S. B. Corder, Modelling the rapid near-surface expansion of gas slugs in low-viscosity magmas, in Fluid Motion in Volcanic Conduits: A Source of Seismic and Acoustic Signals, edited by S. J. Lane and J. S. Gilbert (Geological Society, London, 2008), pp. 147-167.

[11] M. R. James, S. J. Lane, L. Wilson, and S. B. Corder, Degassing at low magma-viscosity volcanoes: Quantifying the transition between passive bubble-burst and Strombolian eruption, J. Volcan. Geotherm. Res. 180, 81 (2009).

[12] R. Kawaguchi and T. Nishimura, Numerical investigation of temporal changes in volcanic deformation caused by a gas slug ascent in the conduit, J. Volcan. Geotherm. Res. 302, 1 (2015).

[13] J. von der Lieth and M. Hort, Slug ascent and associated stresses during Strombolian activity with nonNewtonian rheology, J. Geophys. Res. Solid Earth 121, 4923 (2016).

[14] D. T. Dumitrescu, Strömung an einer Luftblase im senkrechten Rohr, Z. Angew. Math. Mech. 23, 139 (1943).

[15] R. M. Davies and G. I. Taylor, The mechanics of large bubbles rising through extended liquids and through liquids in tubes, Proc. R. Soc. London 200, 375 (1950).

[16] J. Fabre and A. Liné, Modeling of two-phase slug flow, Annu. Rev. Fluid Mech. 24, 21 (1992).

[17] Z.-S. Mao and A. E. Dukler, The motion of Taylor bubbles in vertical tubes. II. Experimental data and simulations for laminar and turbulent flow, Chem. Eng. Sci. 46, 2055 (1991).

[18] E. W. Llewellin, E. del Bello, J. Taddeucci, P. Scarlato, and S. J. Lane, The thickness of the falling film of liquid around a Taylor bubble, Proc. R. Soc. London A 468, 1041 (2012).

[19] A. O. Morgado, J. M. Miranda, J. D. P. Araujo, and J. B. L. M. Campos, Review on vertical gas-liquid slug flow, Int. J. Multiphase Flow 85, 348 (2016).

[20] F. Viana, R. Pardo, R. Yanez, J. L. Trallero, and D. D. Joseph, Universal correlation for the rise velocity of long gas bubbles in round pipes, J. Fluid Mech. 494, 379 (2003).

[21] AAPGWIKI, Drilling fluid. https://wiki.aapg.org/Drilling_fluid (2019).

[22] PetroWIKI, Oil viscosity. https://petrowiki.org/Oil_viscosity (2019).

[23] J. D. Bugg, K. Mack, and K. S. Rezkallah, A numerical model of Taylor bubbles rising through stagnant liquids in vertical tubes, Int. J. Multiphase Flow 24, 271 (1998).

[24] T. Hibiki and M. Ishii, One-dimensional drift-flux model and constitutive equations for relative motion between phases in various two-phase flow regimes, Int. J. Heat Mass Transfer 46, 4935 (2003). 